\title{
Abundancia, producción de materia seca y valor nutricional de leguminosas arbustivas forrajeras del trópico seco
}

\section{Abundance, dry matter production and nutritional value of forage shrub legumes from the dry tropics}

\section{Juan de Dios Guerrero- \\ Rodriguez ${ }^{1 *}$, \\ Angel Bustamante-González 10 , Ernesto Aceves-Ruiz 1 , \\ Samuel Vargas-López ${ }^{1}$ (]) \\ Francisco \\ Calderón- \\ Sánchez' $[$, \\ Efrain Pérez-Ramírez 10 \\ José Isabel Olvera- \\ Hernández 1 [@}

${ }^{1}$ Colegio de Postgraduados, Campus Puebla. Boulevard Forjadores de Puebla No. 205, CP. 72760 . Santiago Momoxpan, San Pedro Cholula, Puebla, México.

${ }^{*}$ Autor de correspondencia: grjuan2000mx@yahoo.com

Artículo científico

Recibido: 24 de febrero 2021

Aceptado: 04 de septiembre 2021

Como citar: Guerrero-Rodríguez JD, Bustamante-González

Aceves-Ruíz E, Vargas-López S, Calderón-Sánchez F, PérezRamírez E, Olvera-Hernández JI (2021) Abundancia, producción de materia seca y valor nutricional de leguminosas arbustivas forrajeras del trópico seco. Ecosistemas y Recursos Agropecuarios Núm. Esp. II: e2881. DOI: 10.19136/era.a8nll.2881
RESUMEN. La selva baja caducifolia tiene amplia diversidad de especies leguminosas arbóreas útiles como alimento para rumiantes, de las cuales se conoce poco. Por tanto, el objetivo fue evaluar siete especies preferidas por los caprinos, para determinar su frecuencia en el agostadero, producción de materia seca de hoja y la calidad nutritiva. La frecuencia relativa en el agostadero se cuantificó mediante cuadrantes de $20 \times 20 \mathrm{~m}$. Con mediciones de la estructura de las plantas, en cada especie se generaron modelos de regresión para la predicción de producción de materia seca de hoja y cuantificación de proteína cruda (PC), fibras y digestibilidad in vitro. La frecuencia relativa varió considerablemente entre especies $(p<0.01)$; Acacia cymbispina fue más abundante. En la producción de materia seca de hoja, las características del tallo principal y de copa del árbol, tuvieron mayor aportación. Las especies difirieron $(p<0.01)$ en concentración de $P C$, situándose en promedio entre 13.2 a 20.6\%, con tendencia a disminuir en Mimosa polyantha, Senna wislizeni y Lysiloma tergemina a medida que progresó el ciclo de crecimiento. Aunque las especies fueron diferentes en la concentración de fibras $(p<0.01)$, en fibra detergente ácido se observaron diferencias mayores, variando de 14.8 a $28.7 \%$, aspecto correlacionado negativamente con la digestibilidad in vitro. La especie más abundante fue $A$. cymbispina, la de mayor concentración de PC fue Acacia farnesiana, la más digestible fue $S$. wislizeni, y la que tuvo menor concentración en PC y digestibilidad fue Acacia milibekii.

Palabras clave: Acacias, agostadero, calidad de forraje, Coursetia glandulosa, ecuaciones alométricas.

ABSTRACT. The dry forest has a wide diversity of species of tree legumes useful as feed for ruminants, of which little is known. Therefore, the objective was to evaluate seven species that goats prefer the most, to determine their frequency in the pasture, production of leaf dry matter and nutritional quality. The relative frequency in the pasture was quantified using $20 \times 20 \mathrm{~m}$ quadrants. With measurements of the plant structure in each species, regression models were generated for the prediction of the leaf dry matter production, crude protein (CP), fiber and in vitro digestibility were quantified. The relative frequency varied considerably between species $(p<0.01)$; Acacia cymbispina was more abundant. In the dry matter production of the leaf, the characteristics of the main stem and the tree's crown had a greater contribution to its estimation. The species differed $(p<0.01)$ in CP concentration, averaging between 13.2 to $20.6 \%$ and with a tendency to decrease in Mimosa polyantha, Senna wislizeni and Lysiloma tergemina as the growth cycle progressed. Although the species were different in fiber concentration $(p<0.01)$, in acid detergent fiber greater differences were observed, varying from 14.8 to $28.7 \%$, an aspect negatively correlated with in vitro digestibility. The most abundant species was A. cymbispina. The species with the highest concentration of CP was Acacia farnesiana, the most digestible was $S$. wislizeni, and the one with the lowest protein concentration and digestibility was Acacia milibekii.

Key words: Acacias, pasture, forage quality, Coursetia glandulosa, allometric equations. 


\section{INTRODUCCIÓN}

Existe una amplia diversidad de especies arbustivas y arbóreas de diferentes tipos de vegetación que se utilizan en la alimentación de rumiantes alrededor del mundo (González-Pech et al. 2015, Nsubuga et al. 2020, Castro et al. 2021). Uno de estos tipos de vegetación es la selva baja caducifolia que se distribuye en varios continentes, principalmente en África, Asia, Oceanía y América (Trejo y Dirzo 2000, Kushwaha et al. 2011). En México, la selva baja caducifolia representa alrededor del $60 \%$ de la vegetación tropical del país (Kauffman et al. 2003, Burgos y Maass 2004), en la cual se tiene una diversidad florística alta y un número considerable de especies endémicas (Guízar-Nolasco et al. 2010, Kushwaha et al. 2011). Entre las familias botánicas que predominan está la Leguminosae (Trejo y Dirzo 2000, Guízar-Nolasco et al. 2010), de la cual, varias de sus especies son aprovechadas para la alimentación del ganado caprino que las ramonea durante la estación de crecimiento, o consumen sus vainas en la época de sequía.

En México, son varios los estudios realizados en relación a la calidad nutritiva de arbustos y árboles para la alimentación de rumiantes, en los cuales se pueden citar los de la selva mediana subperennifolia (Sosa-Rubio et al. 2000, Sosa-Rubio et al. 2004), los de bosque mesófilo, pino-encino de clima templado sub-húmedo (Jimenez-Ferrer et al. 2007, JiménezFerrer et al. 2008) y los de matorral xerófilo (Ramírez y Lara 1998). Para la selva baja caducifolia CarranzaMontaño et al. (2003) y González-Pech et al. (2015) reportaron un número importante de especies consumidas por los rumiantes; sin embargo, existen otras más que son aprovechadas, de las que se conoce poco en abundancia, rendimiento de forraje y calidad nutritiva. Por lo anterior, el objetivo del presente estudio fue determinar de siete leguminosas arbustivas de la selva baja caducifolia en la región "Montaña" de Guerrero, México, la presencia en agostaderos, la producción de materia seca de hoja y su calidad nutritiva, durante la estación de crecimiento.

\section{MATERIALES Y MÉTODOS}

\section{Localización}

El área de estudio se ubicó en la selva baja caducifolia de la región Montaña del estado de Guerrero, México. Las coordenadas del área en mención son $17^{\circ} 50.059^{\prime} \mathrm{LN}$ y $98^{\circ} 31.670^{\prime} \mathrm{LO}$ a17 $31.936^{\prime} \mathrm{N}$ y $98^{\circ} 38.785^{\prime} \mathrm{O}$. El clima es cálido subhúmedo $\left[\mathrm{Aw}_{0}(\mathrm{w})\right]$, con temperatura media anual que varía entre 22 a $26^{\circ} \mathrm{C}$; las lluvias ocurren en el verano y alcanzan anualmente entre 700 a $1200 \mathrm{~mm}$, el cociente precipitación/temperatura es menor a 43.2, la precipitación invernal es menor al $5 \%$ y se presentan marcados periodos de estiaje (Trejo 1999, García 2004). Con el uso de cartografía digital y ortofotos se delimitaron ocho sitios representativos de muestreo, de los cuales, tres quedaron incluidos en el municipio de Tlapa de Comonfort ( $17^{\circ} 31.936^{\prime} \mathrm{LN}, 98^{\circ} 38.785^{\prime}$ LO, 1112 msnm; $17^{\circ} 33.757^{\prime}$ LN, $98^{\circ} 30.161^{\prime}$ LO, 1358 msnm; $17^{\circ} 33.965^{\prime} \mathrm{LN}, 98^{\circ} 32.008^{\prime} \mathrm{LO}, 1$ $017 \mathrm{msnm}$ ); cuatro en Alpoyeca (17 $36.479^{\prime} \mathrm{LN}$, $98^{\circ} 31.355^{\prime}$ LO, $1240 \mathrm{msnm} ; 17^{\circ} 38.734^{\prime} \mathrm{LN}, 98^{\circ}$ 31.442' LO, 976 msnm; $17^{\circ} 39.500^{\prime} \mathrm{LN}, 98^{\circ} 30.009^{\prime}$ LO, 1076 msnm; 17 45.355' LN, 98 35.011' LO, $924 \mathrm{msnm}$ ), y uno en Xochihuehuetlán ( $17^{\circ} 50.059^{\prime}$ LN, 98³1.670' LO, 1252 msnm).

\section{Especies arbustivas seleccionadas}

Con base a una encuesta realizada a 15 informantes clave, productores de ganado caprino de la zona, se identificaron siete especies de leguminosas arbustivas de mayor preferencia por los caprinos. Las especies fueron Lysiloma tergemina Benth, Acacia milibekii Macbride, Mimosa polyantha Benth, Acacia cymbispina Sprague \& L. Riley (sinónimo $A$. cochliacantha), Senna wislizeni Pringlei (Rose) Irwin \& Barneby, Coursetia glandulosa A. Gray y, Acacia farnesiana L. Willd. Las tres primeras especies son endémicas de la depresión del Balsas en México (Camargo-Ricalde et al. 2004, Guízar-Nolazco et al. 2010), las demás se distribuyen desde la parte sur de Estados Unidos a México, con la excepción de $A$. farnesiana que tiene distribución en otros continentes. 


\section{Material vegetal}

El periodo de colecta de hojas fue de julio a noviembre, momento en que la vegetación permanece verde y es cuando existe mayor cantidad de follaje en los árboles y arbustos. Durante este periodo se realizaron cuatro colectas cada 43 días. En cada sitio se identificaron cuatro plantas por especie, no ramoneadas por el ganado. Las plantas de $C$. glandulosa y $M$. polyantha tuvieron una altura promedio de $2.5 \mathrm{~m}$; las de A. cymbispina, A. farnesiana, $L$. tergemina y $S$. wislizeni tuvieron una altura promedio de $3 \mathrm{~m}$, y las de $A$. milibekii fueron de $4 \mathrm{~m}$ de alto. En cada planta se seleccionaron 12 ramas de la periferia, las cuales fueron defoliadas desde su punta a no más de $30 \mathrm{~cm}$ hacía dentro del dosel. Posteriormente, cada rama fue marcada con cinta adhesiva de colores, utilizando un color distinto en cada ocasión para evitar volver a cosechar la misma rama en el muestreo subsecuente; se incluyeron únicamente hojas con peciolo. El material cosechado de las plantas de cada especie se juntó, formando así, una muestra en cada sitio que se tomó como repetición. Las muestras se secaron en una estufa de aire forzado (Shel Lab, Cornelius, Oregon, USA) a $55{ }^{\circ} \mathrm{C}$ hasta alcanzar peso constante. Posteriormente, las muestras se molieron en un molino ciclónico (FossTecator, Höganäs, Suiza) con criba de $1 \mathrm{~mm}$.

\section{Variables medidas}

A cada una de las muestras se le determinó por duplicado la concentración de proteína cruda (CP), fibra detergente neutro (FDN), fibra detergente ácido (FDA) y digestibilidad in vitro (DIVMS). Para la determinación de nitrógeno se utilizó el método MicroKjeldahl utilizando selenio como catalizador (AOAC 1975); este fue multiplicado por 6.25 para obtener la PC calculada. La determinación de FDN se realizó en un analizador de fibra ANKOM 200/220, utilizando los protocolos de ANKOM Technology (ANKOM 2006), excluyendo el paso con alfa-amilasa y el de acetona. La determinación de FDA se realizó después de la determinación de FDN, en el mismo analizador de fibra con el protocolo para FDA de ANKOM Technology (ANKOM 2006) excluyendo el paso con acetona. La DIVMS fue mediante la técnica de dos eta- pas pepsina-celulasa (Jones y Hayward 1975, Clarke et al. 1982, Klein y Baker 1993). Ambas enzimas fueron adquiridas de la compañía SIGMA-ALDRICH. La pepsina (1:10 000 de mucosa de estómago porcino) fue disuelta en $0.125 \mathrm{~N}$ de ácido clorhídrico a una proporción de $6.66 \mathrm{gL}^{-1}$. La celulasa Onozuka RS de Trichoderma viride ( $\geq 5000$ unidades por $\mathrm{g}$ de sólido) se disolvió en buffer acetato $(4.1 \mathrm{~g}$ de acetato de sodio anhidro y $2.9 \mathrm{ml}$ de ácido acético por litro de agua destilada) manteniendo una proporción celulasa:muestra de 1:100 (Clarke et al. 1982). Se utilizó $0.3 \mathrm{~g}$ de materia seca por muestra colocándose en bolsas ANKOM F57. Primeramente, se realizó la etapa de digestión con pepsina y posteriormente la de la celulasa, durando cada una 48 horas en un incubador de agitación orbital a $50^{\circ} \mathrm{C}$ y a 80 revoluciones por minuto.

Como primera aproximación, para observar la dinámica de producción de materia seca durante el periodo de estudio, se cuantificó la producción promedio de materia seca por rama (12 ramas por planta) de los individuos muestreados para calidad de forraje en cada uno de los sitios de muestreo. Asimismo, se cuantificó la abundancia relativa de las especies de interés en 12 sitios, siguiendo un transecto de $400 \mathrm{~m}$ lineales en los que se ubicaron tres puntos aleatorios y en cada uno se colocó un cuadrante de 20x20 m para el conteo de las especies.

Con la finalidad de estimar la producción de materia seca de hoja de un agostadero a través de modelos de regresión, a finales del verano, una vez que la vegetación alcanzó el máximo crecimiento, por especie involucrada en el estudio se tomaron 11 plantas de diferentes alturas a las que los caprinos acostumbran a ramonear. A éstas se les realizaron mediciones en las que se incluyó primeramente la altura $(\mathrm{m})$ de planta. Del tallo se midió el perímetro $(\mathrm{cm})$ de la base, diámetro $(\mathrm{mm})$ de la base, diámetro $(\mathrm{mm})$ de la rama principal en la inserción de la primera, segunda y tercera rama secundaria, así como, el diámetro $(\mathrm{mm})$ de la rama secundaria en la inserción con la principal (estos diámetros básicamente en $M$. polyantha y $S$. wislizeni), altura $(\mathrm{m})$ a la bifurcación del tallo principal (en M. polyantha), diámetro (mm) del tallo principal en su primer bifurcación (en $A$. 
milibekii). De la copa se incluyeron el ancho mayor, el menor y el promedio $(\mathrm{m})$, altura $(\mathrm{cm})$ al vértice inferior de la copa (en $A$. farnesiana), y número de ramas secundarias. Las plantas posteriormente se deshojaron manualmente para determinar la materia seca en una estufa de aire forzado a $100{ }^{\circ} \mathrm{C}$ durante 72 horas.

\section{Análisis estadístico}

El análisis estadístico entre especies se llevó a cabo utilizando el modelo $Y_{i j}=\mu+\beta_{j}+\tau_{i}+\varepsilon_{i j}$ para el análisis por corte y, el $\mathrm{Y}_{i j k}=\mu+\beta_{j}+\mathrm{C}_{j}+$ $\delta_{j i}+\mathrm{V}_{K}+(\mathrm{CV})_{i k}+\varepsilon_{i j k}$ para el análisis combinado de cortes. Para el primer modelo sus términos fueron: $Y_{i j}=$ Variable de interés, $\mu=$ Media poblacional, $\beta_{j}=$ Efecto del sitio j, $\tau_{i}=$ Efecto de la especie y, $\varepsilon_{i j}=$ Error aleatorio ij. Para el modelo lineal del análisis conjunto de todos los cortes los términos fueron: $Y_{i j k}=$ Variable de interés, $\mu=$ Media poblacional, $\beta_{i}=$ Efecto del i-ésimo sitio, $\mathrm{C}_{j}=$ Efecto de corte en su nivel $\mathrm{i}$ (parcela grande), $\delta_{j i}=$ Error asociado del corte, $\mathrm{V}_{K}$ = Efecto de la especie en su nivel $\mathrm{k}$ (parcela chica), $(\mathrm{CV})_{i k}=$ Efecto de la interacción corte*especie al nivel $\mathrm{j}, \mathrm{k} ; \mathrm{y}, \varepsilon_{i j k}=$ Error aleatorio.

Dentro de cada especie para determinar diferencias estadísticas entre cortes, el modelo utilizado fue el mismo que el primero, considerando como $\tau_{i}$ a los cortes.

Para las variables de calidad y producción de materia seca, se hicieron análisis de varianza con el procedimiento GLM; las pruebas de comparación de medias se realizaron con la prueba de Tukey $\operatorname{con} \alpha$ $=0.05$ mediante el paquete estadístico SAS (2009). La frecuencia de las especies se analizó mediante la prueba de Kruskal-Wallis. Para cada especie se obtuvo una ecuación de regresión múltiple para predecir la producción de materia seca de hoja mediante las diferentes mediciones de las plantas, para ello, se utilizó el procedimiento Stepwise de SAS, y se seleccionó la ecuación con mayor $\mathrm{R}^{2}$.

\section{RESULTADOS}

La presencia de las especies en estudio en los agostaderos fue diferente $(p<0.01)$. La de mayor presencia fue $A$. cymbispina en un $94 \%$ de los sitios de muestreo; le siguió $M$. polyantha con $66 \%$; S. wislizeni, L. tergemina, A. milibekii y A. farnesiana se ubicaron en un rango del 41 a $30 \%$, respectivamente, mientras que $\mathrm{C}$. glandulosa estuvo presente sólo en el $16 \%$ de los sitios. En cuanto a la abundancia relativa, A. cymbispina tuvo mayor número de individuos que representaron en promedio el $20 \%$ de la población total por sitio; el número de individuos de $M$. polyantha representó el $8 \%$, en tanto las demás especies estuvieron en el rango de 1 a $5 \%$.

En producción de materia seca por rama de $30 \mathrm{~cm}$ las especies fueron diferentes $(p<0.01)$. La especie que tuvo mayor producción por rama fue $L$. tergemina (Figura 1), mientras que la que produjo menos fue $S$. wislizeni. Al comparar la producción entre cortes dentro de cada especie, se detectaron cambios importantes en $C$. glandulosa $(p=0.01)$ y en M. polyantha $(p=0.04)$ cuya producción disminuyó al avanzar el periodo de muestreo.

Con relación a la estimación de la producción de materia seca de hoja, los modelos de regresión generados incluyeron variables diferentes para cada una de las especies evaluadas. La cantidad de hoja por planta estuvo relacionada con la altura y el número de ramas sólo en cuatro especies (Tabla 1); no obstante, las características del tallo principal y de copa tuvieron mayor participación en la estimación de dicha variable. Los valores de $\mathrm{R}^{2}$ de las ecuaciones seleccionadas en el estudio fueron mayores al $93 \%$, lo que da certeza en la estimación de materia seca de hoja.

Las especies fueron diferentes $(p<0.01)$ en concentración de FDN (Tabla 2). En promedio, $S$. wislizeni, L. tergemina, A. milibekii y A. farnesiana tuvieron los valores más altos. Dentro de especies, quienes tuvieron variaciones $(p \leq 0.02)$ entre cortes fueron las Acacias y L. tergemina (Figura 2) en las que se registró un aumento $(p<0.05)$ al ir avanzando el ciclo de crecimiento; mientras que en las demás hubo similitudes estadísticas ( $p>0.07$ ).

Las especies fueron diferentes en concentración de FDA $(p<0.01)$, con contrastes importantes entre $A$. milibekii, A. cymbispina y $S$. wislizeni, donde A. milibekii tuvo la mayor concentración (28.7\%), $A$. cymbispina una concentración media (22.1\%) y $S$. 

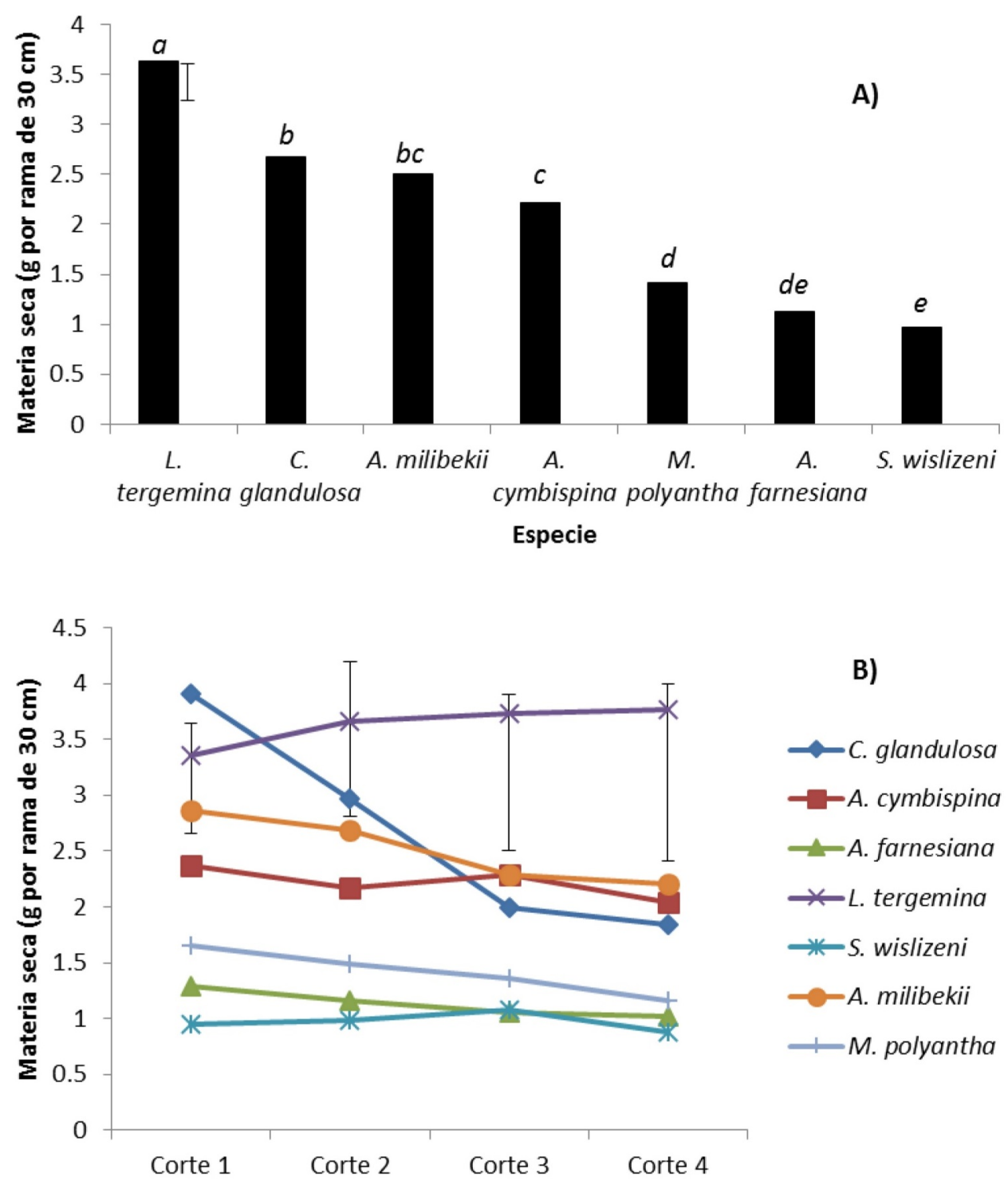

Figura 1. Producción de materia seca por rama de $30 \mathrm{~cm}$ en siete especies de leguminosas arbustivas. A) promedio y B) por corte- aprovechadas como fuente de forraje para caprinos en la región Montaña de Guerrero, México. Las barras representan la diferencia mínima significativa de Tukey $(\alpha=0.05)$ en cada corte para la comparación entre especies.

wislizeni el valor más bajo (14.8\%) (Tabla 2). Dentro de especies, sólo $A$. farnesiana y L. tergemina (Figura 2) tuvieron diferencias entre cortes $(p<0.02)$ tendiendo aumentar a medida que avanzó el ciclo de crecimiento, las demás especies se comportaron sin cambios importantes $(p>0.22)$.

La concentración de PC entre las especies en cada uno de los cortes (Figura 3$)$ fue diferente $(p<$ 0.01 ). La especie con mayor concentración fue $A$. far- nesiana, mientras que $A$. milibekii tuvo las concentraciones más bajas (Tabla 2). Estas dos especies junto con $A$. cymbispina y $C$. glandulosa mantuvieron la concentración de PC durante el periodo de estudio. Sin embargo, M. polyantha, S. wislizeni y $L$. tergemina tuvieron cambios importantes $(p \leq 0.0004)$ a medida que avanzó el ciclo de crecimiento, disminuyendo $(p \leq 0.05)$ de 18.2 a $12.5 \%$ en promedio (Figura 3). 
Tabla 2. Concentración de Proteína cruda, Fibra detergente neutro, Fibra detergente ácido y Digestibilidad in vitro en leguminosas arbustivas, promedio de cuatro cortes.

\begin{tabular}{lcccc}
\hline Especie & $\mathrm{PC}(\%)$ & $\mathrm{FDN}(\%)$ & $\mathrm{FDA}(\%)$ & DIV (\%) \\
\hline Acacia milibekii & $13.2^{d}$ & $41.0^{a}$ & $28.7^{a}$ & $48.9^{f}$ \\
Acacia cymbispina & $17.3^{b}$ & $34.7^{c}$ & $22.1^{c}$ & $52.5^{e}$ \\
Acacia farnesiana & $20.6^{a}$ & $40.1^{a b}$ & $26.0^{b}$ & $60.6^{d}$ \\
Coursetia glandulosa & $15.3^{c}$ & $35.5^{b c}$ & $23.4^{c}$ & $67.3^{c}$ \\
Lysiloma tergemina & $15.4^{c}$ & $41.1^{a}$ & $26.1^{b}$ & $63.1^{d}$ \\
Mimosa polyantha & $16.6^{b c}$ & $31.7^{c}$ & $19.1^{d}$ & $75.6^{b}$ \\
Senna wislizeni & $16.0^{b c}$ & $42.2^{a}$ & $14.8^{e}$ & $83.6^{a}$ \\
& & & & \\
Coeficiente de Variación & 12.5 & 16.0 & 9.0 & 5.2 \\
$\mathrm{R}^{2}$ & 0.81 & 0.73 & 0.93 & 0.96 \\
Especie (P > F) & $<0.0001$ & $<0.0001$ & $<0.0001$ & $<0.0001$
\end{tabular}

Literales en superíndice diferentes dentro de cada columna son estadísticamente diferentes de acuerdo a la prueba de Tukey $(\alpha=0.05)$.

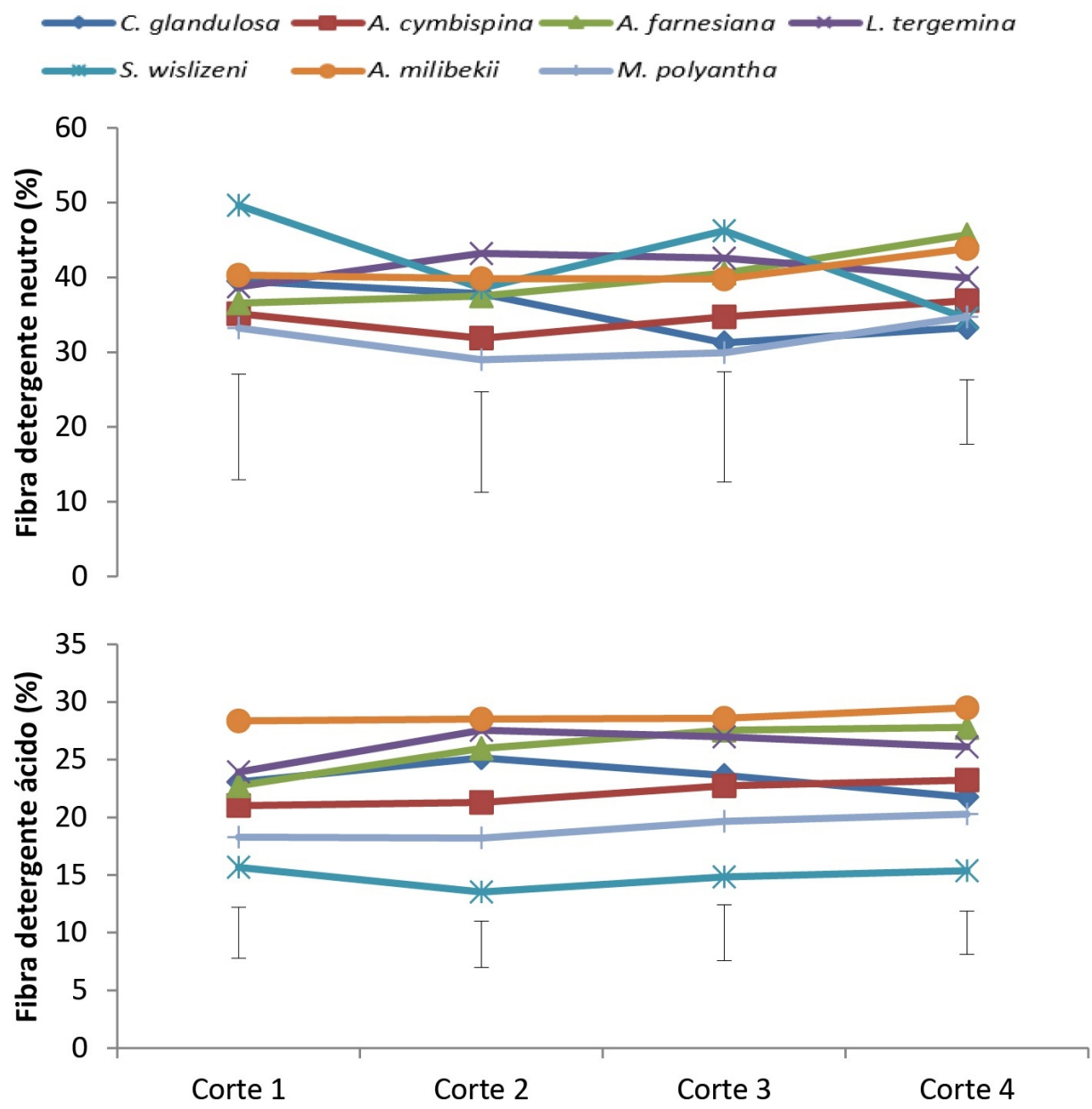

Figura 2. Concentración de Fibra detergente neutro y Fibra detergente ácido, Proteína cruda y Digestibilidad in vitro en siete especies de leguminosas arbustivas aprovechadas como fuente de forraje para caprinos en la región Montaña de Guerrero, México. Las barras representan la diferencia mínima significativa de Tukey $(\alpha=$ 0.05) en cada corte para la comparación entre especies. 

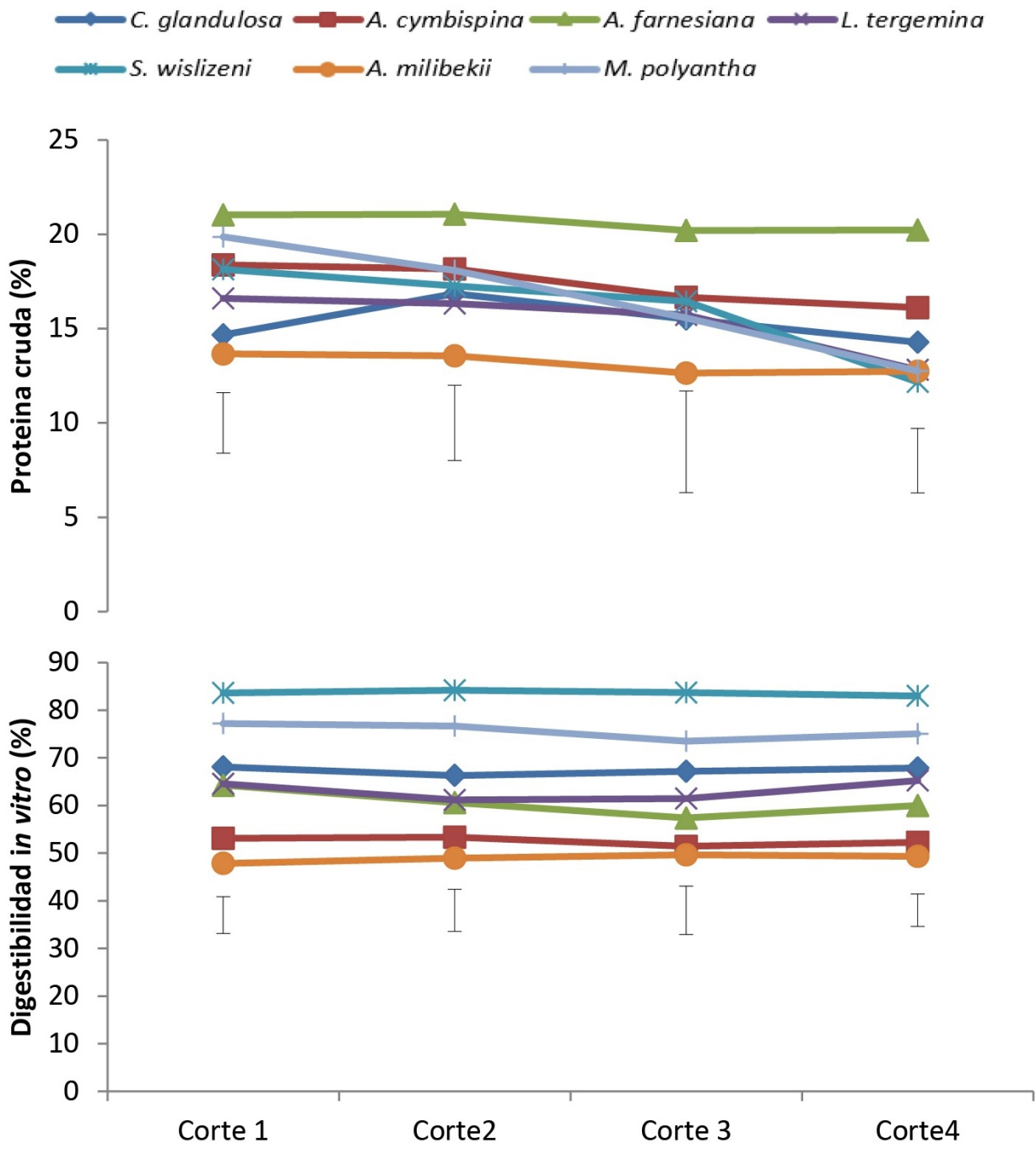

Figura 3. Concentración de Proteína cruda y Digestibilidad in vitro en siete especies de leguminosas arbustivas aprovechadas como fuente de forraje para caprinos en la región Montaña de Guerrero, México. Las barras representan la diferencia mínima significativa de Tukey $(\alpha=0.05)$ en cada corte para la comparación entre especies.

en el Sur del Estado de México, tanto en agostaderos como praderas establecidas, atribuyendo la dispersión a la inclinación del terreno. No obstante, de esta especie se consumen las vainas en abundancia por los rumiantes, lo cual puede ser un factor de mayor dispersión y por tanto de mayor establecimiento. De igual manera ocurre para $A$. Farnesiana, las vainas caen enteras al suelo y son consumidas, no obstante hay varios barrenadores que consumen las semillas (Vergara-Pineda et al. 2014), que de esta manera impiden quizá un mayor establecimiento. En las otras especies, las vainas tienen dehiscencia y cae solamente la semilla, situación que puede ser menos favorable para el establecimiento.

En la producción de materia seca de hoja, las diferencias se debieron principalmente a las características físicas de la hoja entre las especies; por ejemplo, L. tergemina tiene hojas más grandes que S. wislizeni y esta última a su vez tampoco tiene crecimientos exuberantes, características que hicieron que la primera produjera más y la última menos. En lo que se refiere a $A$. farnesiana y $A$. 
cymbispina diferencias en crecimiento se han reportado para plantas en la etapa de desarrollo inicial hasta los 165 días de edad después de la germinación (Cervantes et al. 1998). Estos autores encontraron que $A$. cymbispina desacelera su tasa de crecimiento relativo a medida que avanzó el tiempo el cual se ajustó al periodo de lluvias; dicha situación no se logró detectar en la presente investigación, probablemente por haber sido plantas de mayor edad a las que estudiaron Cervantes et al. (1998). Con respecto a $C$. glandulosa y en $M$. polyantha se observó que fueron las especies que comenzaron la etapa de caída de hoja más temprano, lo que ocasionó a que contrastaran aún más entre cortes. Con base en ello, en este estudio preliminar, se deja ver que existen fluctuaciones en producción de materia seca de hoja entre especies, por lo que estudios posteriores en su dinámica de crecimiento pueden ser de gran utilidad.

En la estimación de la producción de materia seca de hoja las diferencias estructurales y de formas entre especies fueron determinantes. Por ejemplo, en las especies $S$. wislizeni, y $M$. polyantha, que son de copa más amplia, las variables de altura y el número de ramas no funcionaron para la predicción. De igual manera fue con $A$. farnesiana cuyo crecimiento es diferente a todas las demás. Las especies que presentaron mayor similitud en formas de crecimiento fueron $A$. cymbispina, L. tergemina y C. glandulosa en las que las variables de altura, número de ramas y algunas características del tallo y la copa, funcionaron mejor en la predicción.

En cuanto a las variables relacionadas con el valor nutritivo, comenzando con la concentración de proteína cruda, se observó que los valores fueron altos, no obstante, existieron disminuciones que alcanzaron los límites marginales. Los valores menores de $\mathrm{PC}$ encontrados en las últimas etapas de crecimiento (12.5\%) en L. wislizeni, M. polyantha y L. tergemina, así como la baja concentración en $A$. milibekii en todo el periodo, de acuerdo al NRC (1981), escasamente cubrirían los requerimientos en cabritos en crecimiento con alta actividad (i.e., animales de 20 $\mathrm{kg}$ con ganancias diarias de peso de $100 \mathrm{~g}$ y un consumo del $3.5 \%$ de su peso vivo requieren alrededor de $13.1 \%$ de PC) o en cabras lactantes (i.e., animales de $40 \mathrm{~kg}$ produciendo leche con $2.5 \%$ de grasa y consumo de $3 \%$ de su peso vivo requieren $13.9 \%$ de $\mathrm{PC}$ ), condición que se complica al disminuir la producción de materia seca. Sin embargo, algunos autores mencionan que existe reciclado de nitrógeno en el que el tipo de animal (salvajes y del desierto) y la dieta (altas en taninos) compensan los requerimientos de PC (Silanikove 2000, Sanon et al. 2008). En A. farnesiana el intervalo de PC reportado por algunos autores en un sólo momento del ciclo vegetativo va de 20 a 24\% (Ramírez y Ledezma 1997, Ramírez y Lara 1998, Benavides 1999, Ramírez et al. 2000, Pinto et al. 2002, Carranza-Montaño et al. 2003), intervalo en el que se ubicaron los valores encontrados en la presente investigación. De acuerdo con la Figura 2, A. farnesiana mantiene concentraciones estables y es probable que se mantengan iguales a través de ambientes. Para A. cymbispina, Carranza-Montaño et al. (2003) reportan $16.9 \%$ de PC, valor no muy alejado del encontrado en la presente investigación (Tabla 2); en las demás especies no se tiene referencia alguna de esta variable, ni para las demás.

Las diferencias entre especies en la concentración promedio de FDN son comunes como se reporta para las especies que estudiaron Sosa et al. (2004). Valores de referencia de FDN, sólo se tienen para $A$. farnesiana los cuales se reportan en el intervalo de 37.7 a 42.1\% (Ramírez y Ledezma 1997, Ramírez y Lara 1998, Ramírez et al. 2000, Pinto et al. 2002), en el cual se ubica el valor encontrado en la presente investigación.

Para FDA así mismo, sólo se tienen reportes para $A$. farnesiana en el intervalo de 23.3 a $26.7 \%$ (Ramírez y Ledezma 1997, Ramírez y Lara 1998, Ramírez et al. 2000, Pinto et al. 2002), en el cual también se ubica el valor encontrado en la presente investigación. En esta fracción de fibra se nota que las especies tienen diferencias en la estructura de la pared celular, probablemente en el tipo y cantidad de hemicelulosas y algunos otros compuestos, al encontrarse que $A$. milibekii y $S$. wislizeni o A. cymbispina y $M$. polyantha tuvieron concentraciones similares de FDN, pero difieren en FDA. En general, Lu et al. (2005) mencionan que concentraciones entre $18 \mathrm{a}$ 
$23 \%$ de FDA pueden ser adecuadas nutricionalmente para cabras en lactación y en crecimiento, por lo que al menos cuatro de estas especies (Tabla 1) pueden cumplir este parámetro.

Las diferencias encontradas en digestibilidades es posible que se deban a la variación morfológica de las especies y de composición química de la pared celular. Por ejemplo, S. wislizeni tiene hojas pequeñas de peciolo corto y nervaduras delgadas; $A$. milibekii tiene hojas más grandes un tanto coriáceas, lo cual puede influenciar los valores de digestibilidad. Así también, no todas las especies acumulan los mismos compuestos fibrosos.

Datos referentes a digestibilidad in vitro para A. farnesiana son reportados por Benavides (1999) y por Pinto et al. (2002), quienes encontraron valores de 41.7 y $55.0 \%$, respectivamente. Los valores encontrados en la presente investigación son más elevados y dan evidencia que esta especie presenta mayor variación que algunas otras de las especies evaluadas. La digestibilidad que presentó $A$. milibekii es considerada marginal para la producción animal y probablemente la de $A$. cymbispina que están por mitad.

Entre las especies, $A$. farnesiana, aun cuando fue la que mayor contenido de proteína cruda presentó, y esta concentración se mantiene estable, tiene una digestibilidad media, produce poca materia seca, es espinosa y su frecuencia relativa es baja. A. cymbispina presenta buen contenido de $\mathrm{PC}$, la cual se mantiene estable por un tiempo prolongado; comparada con las demás especies, tiene una producción media por rama y una digestibilidad baja, pero es abundante. La especie $M$. polyantha tiene una aceptable concentración de PC, pero dicha concentración decrece conforme avanza la estación de crecimiento, produce poca materia seca la cual también decrece, es espinosa, pero presenta alta digestibilidad y tiene una frecuencia relativa media. La especie S. wislizeni su concentración de PC es también aceptable, aunque tiende a decrecer con el tiempo, tiene poca producción de materia seca, su frecuencia relativa es regular, pero tiene alta digestibilidad. Por su parte, C. glandulosa tiene aceptable concentración de PC que se mantiene a través del tiempo, tiene una digestibilidad media, su producción de materia seca se encuentra en un término medio, no tiene espinas, pero disminuye su producción rápidamente y su frecuencia es baja. La especie L. tergemina tiene una aceptable concentración de PC, pero decrece a medida que avanza el tiempo, tiene una digestibilidad media, no tiene espinas, su frecuencia relativa es regular y produce más materia seca por rama que las demás especies. La especie M. milibekii aunque su concentración de $\mathrm{PC}$ es estable durante el tiempo, ésta tiene bajo contenido, tiene una producción media por rama, es espinosa, su presencia es baja y tiene una digestibilidad baja; sin embargo, es de las pocas especies que permanecen con hojas por mayor tiempo.

\section{CONCLUSIONES}

Las especies estudiadas son diferentes en concentración de proteína cruda, digestibilidad, producción de materia seca y frecuencia relativa en el agostadero. La especie con mayores concentraciones de proteína cruda fue $A$. farnesiana, la más digestible fue $S$. wislizeni, la más abundante fue $A$. cymbispina y la que tuvo menor calidad en proteína y digestibilidad fue $A$. milibekii.

\section{LITERATURA CITADA}

AOAC (1975) Official Methods of Analysis of the Association of Official Agricultural Chemists. AOAC International. Washington, D.C. USA. 1094p.

ANKOM Technology (2006) Operator's Manual ANKOM ${ }^{200 / 220}$ Fiber Analyzer. ANKOM Technology, Macedon, NY, USA. 21p.

Benavides JE (1999) Árboles y arbustos forrajeros: una alternativa agroforestal para la ganadería. En: Sánchez 
MD, Rosales MM (eds). Agroforestería para la producción animal en Latinoamérica. Memorias de una conferencia electrónica realizada de Abril a Septiembre de 1998, Estudio FAO Producción y Sanidad Animal 143. Roma, Italia. pp. 367-394.

Burgos A, Maass JM (2004) Vegetation change associated with land-use in tropical dry forest areas of Western Mexico. Agriculture, Ecosystems and Environment 104: 475-481.

Camargo-Ricalde SL, Dhillion SS, García-García V (2004) Phenology, and seed production and germination of seven endemic Mimosa species (Fabaceae-Mimosoideae) of the Tehuacán-Cuicatlán Valley, Mexico. Journal of Arid Environments 58: 423-437.

Carranza-Montaño MA, Sánchez-Velásquez LR, Pineda-López MdR, Cuevas-Guzmán R (2003) Calidad y potencial forrajero de especies del bosque tropical caducifolio de la sierra de Manantlán, México. Agrociencia 37: 203-210.

Castro M, Teixeira A, Fernández-Núñez E (2021) The nutritive value of different Mediterranean browse species used as animal feeds under oak silvopastoral systems in Northern Portugal. Agroforestry Systems 95: 269-278.

Cervantes V, Arriaga V, Meave J, Carabias J (1998) Growth analysis of nine multipurpose woody legumes native from southern Mexico. Forest Ecology and Management 110: 329-341.

Clarke T, Flinn PC, McGowan AA (1982) Low-cost pepsin-cellulase assays for prediction of digestibility of herbage. Grass and Forage Science 37: 147-150.

García E (2004) Modificaciones al sistema de clasificación climática de Köppen, para adaptarlo a las condiciones de la República Mexicana. Series Libros No. 6, Instituto de Geografía, UNAM. México. 90p.

González-Pech PG, Torres-Acosta FJ, Sandoval-Castro CA, Tun-Garrido J (2015) Feeding behavior of sheep and goats in a deciduous tropical forest during the dry season: The same menu consumed differently. Small Ruminant Research 133: 128-134.

Guízar-Nolasco E, Granados-Sánchez D, Castañeda-Mendoza A (2010) Flora y vegetación en la porción sur de la Mixteca Poblana. Revista Chapingo Serie Ciencias Forestales y del Ambiente 16: 95-118.

Jiménez-Ferrer G, López-Carmona M, Nahed-Toral J, Ochoa-Gaona S, de Jong B (2008) Árboles y arbustos forrajeros de la región norte-tzotzil de Chiapas, México. Veterinaria México 39: 199-213.

Jiménez-Ferrer G, Pérez-López H, Soto-Pinto L, Nahed-Toral J, Hernández-López L, Carmona de la Torre J (2007) Livestock, nutritive value and local knowledge of fodder trees in fragment landscapes in Chiapas, Mexico. Interciencia 32: 274-280.

Jones DIH, Hayward MV (1975) The effect of pepsin pretreatment of herbage on the prediction of dry matter digestibility from solubility in fungal cellulase solutions. Journal of the Science of Food and Agriculture 26: 711-718.

Kauffman JB, Steele MD, Cummings DL, Jaramillo VJ (2003) Biomass dynamics associated with deforestation, fire, and, conversion to cattle pasture in a Mexican tropical dry forest. Forest Ecology and Management 176: 1-12.

Klein L, Baker SK (1993) Composition of the fractions of dry, mature subterranean clover digested in vivo and in vitro, In: Baker MJ, Crush JR, Humphreys LR (eds). Proceedings of the XVII International Grasslands Congress. New Zealand Grassland Association. Palmerston North, New Zealand. pp: 593-595.

Kushwaha CP, Tripathi SK, Tripathi BD, Singh KP (2011) Patterns of tree phenological diversity in dry tropics. Acta Ecologica Sinica 31: 179-185. 
Leung B, Hudgins EJ, Potapova A, Ruiz-Jaen MC (2019) A new baseline for countrywide $\alpha$-diversity and species distributions: illustration using $>6,000$ plant species in Panama. Ecological Applications 29: 1-13.

Lu CD, Kawas JR, Mahgoub OG (2005) Fiber digestion and utilization in goats. Small Ruminant Research 60: 45-62.

NRC (1981) Nutrient requirements of goats: Angora, Dairy, and meat goats in temperate and tropical countries. National Academy Press. Washington, D. C. USA. 91p.

Nsubuga D, Nampanzira DK, Masembe C, Muwanika VB (2020) Nutritional properties of some browse species used as goat feed in Pastoral dry lands, Uganda. Agroforestry Systems 94: 933-940.

Olivares-Pérez J, Avilés-Nova F, Albarrán-Portillo B, Rojas-Hernández S, Castelán-Ortega O (2011) Identificación, usos y medición de leguminosas arbóreas forrajeras en ranchos ganaderos del sur del Estado de México. Tropical and Subtropical Agroecosystems 14: 739 -748.

Pinto R, Ramírez L, Kú VJC, Ortega L (2002) Especies arbóreas y herbáceas forrajeras del sureste de México. Pastos y Forrajes 25: 171-180.

Ramírez RG, Lara JA (1998) Influence of native shrubs Acacia rigidula, Cercidium macrum and Acacia farnesiana on digestibility and nitrogen utilization by sheep. Small Ruminant Research 28: 39-45.

Ramírez RG, Ledezma-Torres RA (1997) Forage utilization from native shrubs Acacia rigidula and Acacia farnesiana by goats and sheep. Small Ruminant Research 25: 43-50.

Ramírez RG, Neira-Morales RR, Ledezma-Torres RA, Garibaldi-González CA (2000) Ruminal digestion characteristics and effective degradability of cell wall of browse species from northeastern Mexico. Small Ruminant Research 3: 49-55.

Sanon HO, Kaboré-Zoungrana C, Ledin I (2008) Nutritive value and voluntary feed intake by goats of three browse fodder species in the Sahelian zone of West Africa. Animal Feed Science and Technology 144: 97-110.

SAS (2009) SAS Institute. SAS/STAT $B$ 9.2. User Guide Release. Cary, NC: SAS Institute Inc. USA. 60p.

Silanikove N (2000) The physiological basis of adaptation in goats to harsh environments. Small Ruminant Research 35: 181-193.

Sosa REE, Pérez RD, Ortega RL, Zapata BGdJ (2004) Evaluación del potencial forrajero de árboles y arbustos tropicales para la alimentación de ovinos. Técnica Pecuaria en México 42: 129-144.

Sosa REE, Sansores LLI, Zapata BGdJ, Ortega RL (2000) Composición botánica y valor nutricional de la dieta de bovinos en un área de vegetación secundaria en Quintana Roo. Técnica Pecuaria en México 38: 105-117.

Trejo I, Dirzo R (2000) Deforestation of seasonally dry tropical forest: a national and local analysis in Mexico. Biological Conservation 94: 133-142.

Trejo VI (1999) El clima de la selva baja caducifolia en México. Investigaciones Geográficas 39: 40-52.

Vergara-Pineda S, Jones RW, Malda-Barrera G, Caltzontzin-Fernández K, Obregón-Zúñiga A, Cambrón-Sandoval VH (2014) Coelocephalapion subornatum (Fall) (Coleoptera: Brentidae: Apioninae) forms galls in stems of Acacia farnesiana (L.) Willd. in Central Mexico. Southwestern Entomologist 40: 223-226. 